\title{
Zero balance ultrafiltration using dialysate during nationwide bicarbonate shortage: a retrospective analysis
}

\author{
Ryan Mullane ${ }^{1 *}$ D, Lance Fristoe ${ }^{2}$, Nicholas W. Markin ${ }^{3}$, Tara R. Brakke ${ }^{3}$, Helen Mari Merritt-Genore ${ }^{4}$,
} Aleem Siddique $^{4}$, Clifford D. Miles ${ }^{1}$ and Troy J. Plumb ${ }^{1}$

\begin{abstract}
Background: Zero balance ultrafiltration (Z-BUF) utilizing injectable 8.4\% sodium bicarbonate is utilized to treat hyperkalemia and metabolic acidosis associated with cardiopulmonary bypass (CPB). The nationwide shortage of injectable 8.4\% sodium bicarbonate in 2017 created a predicament for the care of cardiac surgery patients. Given the uncertainty of availability of sodium bicarbonate solutions, our center pro-actively sought a solution to the sodium bicarbonate shortage by performing Z-BUF with dialysate (Z-BUF-D) replacement fluid for patients undergoing cardiopulmonary bypass.
\end{abstract}

Methods: Single-center, retrospective observational evaluation of the first 46 patients at an academic medical center who underwent Z-BUF using dialysate over a period of 150 days with comparison of these findings to a historical group of 39 patients who underwent Z-BUF with sodium chloride (Z-BUF-S) over the preceding 150 days. The primary outcome was the change in whole blood potassium levels pre- and post-Z-BUF-D. Secondary outcomes included changes in pre- and post-Z-BUF-D serum bicarbonate levels and the amount of serum bicarbonate used in each Z-BUF cohort (Z-BUF-D and Z-BUF-S).

Results: Z-BUF-D and Z-BUF-S both significantly reduced potassium levels during CPB. However, Z-BUF-D resulted in a significantly decreased need for supplemental $8.4 \%$ sodium bicarbonate administration during CPB $(52 \mathrm{mEq} \pm 48$ vs. $159 \mathrm{mEq} \pm 85, P<0.01)$. There were no complications directly attributed to the Z-BUF procedure.

Conclusion: Z-BUF with dialysate appears to be analternative to Z-BUF with sodium chloride with marked lower utilization of intravenous sodium bicarbonate.

Keywords: Zero balance ultrafiltration, Z-BUF, Dialysate

\section{Background}

Cardiopulmonary bypass $(\mathrm{CPB})$ can be associated with the development of metabolic derangements, including metabolic acidosis (serum bicarbonate $<22 \mathrm{mEq} / \mathrm{L}$ ) and hyperkalemia (serum potassium $>5.1 \mathrm{mEq} / \mathrm{L}$ ). These derangements occur secondary to lactic acidosis and tissue hypoperfusion, hemodilution and changes in strong ion difference [1]. Hyperkalemia can occur due to hemolysis and renal dysfunction, or as a result of the high-potassium containing

\footnotetext{
* Correspondence: ryan.mullane@unmc.edu

${ }^{1}$ Department of Internal Medicine, University of Nebraska Medical Center, 983040 Nebraska Medical Center, Omaha, NE 68198-3040, USA

Full list of author information is available at the end of the article
}

cardioplegia solution utilized to electrically arrest the myocardium during aortic cross-clamping [2].

Zero-balance ultrafiltration (Z-BUF) is the process by which a solution (replacement fluid) is infused into the $\mathrm{CPB}$ circuit and an equal volume of fluid is removed via an ultrafiltration column. This process allows small molecules to be removed from the blood, while cells and most proteins remain. Z-BUF therefore effectively allows for the removal of substances such as potassium, via convective clearance, and allows for the addition of substances, such as bicarbonate, without a net gain of fluid by the patient. Z-BUF was initially conceptualized to reduce the inflammatory mediators associated with $\mathrm{CPB}$

(C) The Author(s). 2019 Open Access This article is distributed under the terms of the Creative Commons Attribution 4.0 International License (http://creativecommons.org/licenses/by/4.0/), which permits unrestricted use, distribution, and 
[3], though it is now commonly utilized to assist in the correction of electrolyte abnormalities that can occur during $\mathrm{CPB}$.

A national shortage of sodium bicarbonate injections developed in 2017 due to delays in manufacturing [4]. Prior to the sodium bicarbonate shortage, our institution routinely used sodium chloride solutions $(\mathrm{NaCl})[0.9 \%$ sodium chloride and $0.45 \%$ sodium chloride, usually in a 2:1 ratio] as the initial replacement fluids for Z-BUF and concurrently administered intravenous $8.4 \%$ sodium bicarbonate when needed for metabolic acidosis. With the nationwide shortage of sodium bicarbonate, the liberal use of sodium bicarbonate solution to correct metabolic acidosis was curtailed and we thus developed an alternate strategy to correct this abnormality.

Dialysate solutions contain bicarbonate with a relatively physiologic balance of electrolytes when compared to serum. Two single patient cases have been reported using Z-BUF with dialysate replacement fluid to treat both hyperkalemia and metabolic acidosis $[5,6]$. With the extremely reduced supply of sodium bicarbonate, yet ongoing need to perform $\mathrm{CPB}$ at our facility, we elected to perform Z-BUF during $\mathrm{CPB}$ with dialysate solution as the replacement fluid to manage the ensuant hyperkalemia and metabolic acidosis. Herein, we describe our experience using dialysate as the replacement fluid for Z-BUF during CPB for electrolyte management in a large number of patients and show the results of a historical comparator group who underwent Z-BUF with $\mathrm{NaCl}(0.9 \%$ sodium chloride). We hypothesized that the use of dialysate as the replacement fluid for Z-BUF would show similar decreases in whole blood potassium levels and increases in serum bicarbonate levels when compared to Z-BUF with $\mathrm{NaCl}$ as the replacement fluid.

\section{Methods}

Following review of the available published case reports $[5,6]$ regarding the use of dialysate for electrolyte correction via $\mathrm{Z}$-BUF during $\mathrm{CPB}$, the decision to proceed with its use was deemed appropriate by a team comprised of cardiovascular anesthesiology, cardiothoracic surgery, nephrology, perfusion and pharmacy. The utilization of Z-BUF for the specific subjects was determined at the discretion of the clinical perfusionist after consultation with the cardiovascular anesthesiologist and cardiovascular surgeon at the time of the surgical procedure. Z-BUF was usually initiated for patients who had hyperkalemia, metabolic acidosis, or both. Patients undergoing both scheduled and emergent procedures are included in this report. The "Z-BUF dialysate" group (Z-BUF-D) consisted of all patients undergoing $\mathrm{CPB}$ at our single institution in which Z-BUF was performed with dialysate in the first 150 days following switching to the dialysate solution. We decided to review patients within the first 150 days of the change to utilize dialysate as the replacement fluid for Z-BUF as that coincided with the greatest restrictions to sodium bicarbonate administration at our institution. Following approval from our Institutional Review Board (752-17-EP, 11/22/2017), we retrospectively collected patient demographics including age, sex, type of procedure, time spent on $\mathrm{CPB}$ and laboratory values from the electronic medical record. Laboratory testing that was reviewed included pre- and post-Z-BUF whole potassium levels (measured via blood gas analyzers) and pre- and post-Z-BUF bicarbonate levels obtained from venipuncture or indwelling central venous catheters). We also collected data on those patients at our single center who had undergone standard Z-BUF with $\mathrm{NaCl}$ (Z-BUF-S) in the 150 days prior to initiating Z-BUF-D and utilized them as a comparator group.

Patients were perfused using our institution's standard, custom CPB circuit containing a Capiox ${ }^{\circ}$ FX Advanced oxygenator with integrated arterial filter (Terumo Cardiovascular Group, Ann Arbor, MI) primed with $700 \mathrm{ml}(\mathrm{mL})$ of PLASMA-LYTE A Inj. pH 7.4 (Baxter, Deerfield, IL). The Z-BUF was performed using a polysulfone fiber hemoconcentrator (Livanova, London). A $250 \mathrm{~mL} / \mathrm{min}$ blood shunt was diverted off the recirculation line through the hemoconcentrator back to the venous reservoir. Dialysate solution was administered in approximately $500 \mathrm{~mL}$ boluses to maintain a safe venous reservoir level during the Z-BUF procedure. At our institution, Z-BUF is utilized for patients with hyperkalemia, metabolic acidosis, or those patients in whom these disorders are expected to occur because of the time or nature of the procedure. The dialysate solutions utilized were the NxSTAGE Pure Flow ${ }^{\text {ma }}$ Bicarbonate Solutions RFP- $400^{\circ}\left(2 \mathrm{mEq} / \mathrm{L} \mathrm{K}^{+}, 35 \mathrm{mEq} / \mathrm{L} \mathrm{HCO}^{-}{ }_{3}\right)$ and RFP$402^{\circ}\left(0 \mathrm{mEq} / \mathrm{L} \mathrm{K}^{+}, 35 \mathrm{mEq} / \mathrm{L} \mathrm{HCO}^{-}{ }_{3}\right)(\mathrm{NxStage}$, Lawrence, MA) (Table 1), which are supplied as $5 \mathrm{l}$ (L) bags and cost between $\$ 20$ to $\$ 25$ per bag. The type and volume of dialysate incorporated as replacement fluid was determined by the treatment team, with the RFP- $400^{\circ}$ solution used initially due to availability and then a switch to primarily using RFP- $402^{\circ}$ solutions (when they were obtained from NxSta$\left.\mathrm{ge}^{\circ}\right)$ given its lower potassium concentration. Dialysate use as replacement fluid was limited to the duration of $\mathrm{CPB}$ as required by the associated surgical procedure.

Our analysis is a retrospective, observational comparison to a historical cohort. The primary outcome was the change in whole blood potassium levels pre- and post-ZBUF. Secondary outcomes included changes in pre- and post-Z-BUF serum bicarbonate levels and the amount of serum bicarbonate used for Z-BUF. Normally distributed variables are presented as mean value \pm standard deviation. Normal probability plots were created to determine that the data (whole blood potassium and serum bicarbonate) was normal. Student's T test was used to compare the values of the variables between groups. $P<0.05$ was considered an indication of a statistically significant result. 
Table 1 Content of Potential Solutions Used During Z-BUF [7, 8]

\begin{tabular}{|c|c|c|c|c|c|c|c|c|c|}
\hline Product & $\begin{array}{l}\text { Bicarbonate } \\
(\mathrm{mEq} / \mathrm{L})\end{array}$ & $\begin{array}{l}\text { Potassium } \\
\text { (mEq/L) }\end{array}$ & $\begin{array}{l}\text { Sodium } \\
(\mathrm{mEg} / \mathrm{L})\end{array}$ & $\begin{array}{l}\text { Calcium } \\
(\mathrm{mEg} / \mathrm{L})\end{array}$ & $\begin{array}{l}\text { Chloride } \\
(\mathrm{mEg} / \mathrm{L})\end{array}$ & $\begin{array}{l}\text { Glucose } \\
(\mathrm{mg} / \mathrm{dL}) \\
\end{array}$ & $\begin{array}{l}\text { Lactate } \\
(\mathrm{mmol} / \mathrm{L})\end{array}$ & $\begin{array}{l}\text { Acetate } \\
(\mathrm{mmol} / \mathrm{L})\end{array}$ & $\begin{array}{l}\text { Gluconate } \\
(\mathrm{mmol} / \mathrm{L})\end{array}$ \\
\hline RFP-400 & 35 & 2 & 140 & 3 & 111 & 100 & - & - & - \\
\hline RFP-402 ${ }^{\circledast}$ & 35 & 0 & 140 & 3 & 109 & 100 & - & - & - \\
\hline Normal saline (0.9\%) & - & - & 154 & - & 154 & - & - & - & - \\
\hline 0.45\% Sodium Chloride & - & - & 77 & - & 77 & - & - & - & - \\
\hline Lactated Ringer's & - & 5.4 & 131 & 2 & 111 & - & 29 & - & - \\
\hline Plasma-Lyte A & - & 5 & 140 & - & 98 & - & - & 27 & 23 \\
\hline 8.4\% Sodium Bicarbonate & 1000 & - & 1000 & - & - & - & - & - & - \\
\hline
\end{tabular}

\section{Results}

Forty-six patients underwent CPB with Z-BUF-D during the first 150 days following the change in Z-BUF protocol. Thirty-nine patients who had undergone CPB with Z-BUF-S during the 150 days prior to the sodium bicarbonate shortage were reviewed to serve as a reference group. Details regarding these subjects are summarized in Table 2. Of the Z-BUF-D subjects, 30 were male and 16 were female. The mean age of this group was $62.5 \pm$ 12.8 years, mean duration of $\mathrm{CPB} 158 \pm 77 \mathrm{~min}$ and mean baseline creatinine $1.6 \pm 1.6 \mathrm{mg} / \mathrm{dL}$. This cohort included four patients with end-stage renal disease (ESRD) and ten patients with chronic kidney disease stage 3-5. The volume of replacement fluid ranged from $1300 \mathrm{ml}$ $(\mathrm{mL})$ to $10,000 \mathrm{~mL}$. The mean volume of replacement fluid used in Z-BUF-S patients was $2413.16 \mathrm{~mL}$ while the mean volume of dialysate used as a replacement fluid in the Z-BUF-D group was $4428.38 \mathrm{~mL}(P<0.01)$.

Table 2 Characteristics of patients who underwent Z-BUF with and without dialysate as replacement fluid

\begin{tabular}{llll}
\hline Characteristics & $\begin{array}{l}\text { Z-BUF-S } \\
(n=39)\end{array}$ & $\begin{array}{l}\text { Z-BUF-D } \\
(n=46)\end{array}$ & p value \\
\hline Age (years) & $53.1 \pm 13.6$ & $62.5 \pm 12.8$ & $<0.01$ \\
Female sex - number (\%) & $9(23 \%)$ & $16(35 \%)$ & 0.12 \\
Pre-operative creatinine (mg/dL) & $1.9 \pm 2.4$ & $1.6 \pm 1.6$ & 0.25 \\
Pre-operative potassium (mEq/L) & $4.2 \pm 0.5$ & $4.2 \pm 0.4$ & 0.34 \\
Pre-operative bicarbonate (mEq/L) & $26.2 \pm 3$ & $26.0 \pm 3.3$ & 0.38 \\
$\begin{array}{l}\text { Coronary Artery Bypass Grafting } \\
\text { (CABG) - number (\%) }\end{array}$ & $15(38 \%)$ & $23(50 \%)$ & 0.31 \\
Valve Repair - number (\%) & $13(33 \%)$ & $11(24 \%)$ & \\
Aortic Dissection or Aneurysm & $8(21 \%)$ & $8(17 \%)$ & \\
Repair - number (\%) & & & 0.39 \\
Other procedure - number (\%) & $3(8 \%)$ & $4(9 \%)$ & 7 \\
Mortality (\%) & 8 & 7 & \\
\hline
\end{tabular}

Pre-operative potassium levels vary from pre-Z-BUF potassium levels (peak intraoperative). Patients who did not receive dialysate as a replacement fluid were from prior to sodium bicarbonate shortage. Patients who did receive dialysate as a replacement fluid were from after sodium bicarbonate shortage. Plus-minus values are means \pm standard deviation

$Z$-BUF zero balance ultrafiltration

$Z-B U F-S$ zero balance ultrafiltration with sodium chloride solutions

$Z-B U F-D$ zero balance ultrafiltration with dialysate solution
The mean peak intraoperative whole blood potassium level (pre-Z-BUF) was $6.4 \pm 0.73 \mathrm{mEq} / \mathrm{L}$ in the Z-BUF-S group and $5.6 \pm 0.68 \mathrm{mEq} / \mathrm{L}$ in the Z-BUF-D group $(P<0.01)$. The mean post-Z-BUF whole blood potassium level was $4.6 \pm 0.68 \mathrm{mEq} / \mathrm{L}$ in the Z-BUF-S group and $4.3 \pm 0.56 \mathrm{mEq} / \mathrm{L}$ in the Z-BUF-D group. The difference between the pre-Z-BUF and post-Z-BUF potassium was significant in both groups $(P<0.01)$. All patients undergoing Z-BUF-D had a decrease in their whole blood potassium level (with the exception of one patient, for whom information was not available as they died intra-operatively).

The mean pre-Z-BUF bicarbonate level was $26.2 \pm 4.2$ $\mathrm{mEq} / \mathrm{L}$ in the Z-BUF-S group and $25.9 \pm 3.4 \mathrm{mEq} / \mathrm{L}$ in the Z-BUF-D group $(P=0.31)$. The mean post-Z-BUF serum bicarbonate level was $22.1 \pm 2.67 \mathrm{mEq} / \mathrm{L}$ in the Z-BUF-S group and $22.6 \pm 2.37 \mathrm{mEq} / \mathrm{L}$ in the Z-BUF-D group. Overall, there was a decrease in the serum bicarbonate concentration following the $\mathrm{CPB}$ period in all patients undergoing Z-BUF. The change in serum bicarbonate was similar in the patients undergoing Z-BUF-D and Z-BUF-S $(-3.32 \pm 3.96 \mathrm{mEq} / \mathrm{L}$ and $-4.18 \pm 3.59 \mathrm{mEq} / \mathrm{L}, \quad P=0.13)$. The average $8.4 \%$ sodium bicarbonate used per case was significantly less in the patients undergoing Z-BUF-D (52 $\pm 48 \mathrm{mEq} /$ patient) compared to the patients undergoing Z-BUF-S (159 $\pm 85 \mathrm{mEq} /$ patient $)(P<0.01)$.

The post-Z-BUF serum sodium levels were similar between the Z-BUF-S $(142.6 \pm 3.68 \mathrm{mEq} / \mathrm{L})$ and Z-BUF-D cohorts $(141.8 \pm 0.15 \mathrm{mEq} / \mathrm{L}) \quad(P=0.15)$. The post-Z-BUF serum chloride levels were also similar in the Z-BUF-S and Z-BUF-D groups $(111.08 \pm 4.39 \mathrm{mEq} / \mathrm{L}$ versus $109.76 \pm$ $2.84 \mathrm{mEq} / \mathrm{L})(P=0.06)$. The post-Z-BUF $\mathrm{pH}$ and $\mathrm{PaCO} 2$ levels did not differ between the Z-BUF-S and ZBUF-D patients.

Seven percent of the in the Z-BUF-D cohort died, all of complications unrelated to Z-BUF. There was no difference in mortality when compared to the patients undergoing Z-BUF-S $(P=0.39)$. Six patients without pre-existing ESRD required dialysis following their procedures; one patient had been on dialysis for acute kidney injury prior to their procedure and was continued on dialysis thereafter. Of the five patients who had not 
been on dialysis prior to their procedures, one patient required initiation of hemodialysis on post-operative day 1 due to hyperkalemia, one patient required initiation of hemodialysis on post-operative day 0 due to acidemia (due to type A lactic acidosis). One patient required hemodialysis on post-operative day 7 due to anuric renal failure likely secondary to ischemic acute tubular necrosis, one patient required hemodialysis on post-operative day 5 for volume removal and one required hemodialysis on a hospital admission within one month of their procedure.

\section{Discussion}

Hyperkalemia and metabolic acidosis are complications that can occur during CPB. Z-BUF-S is effective at lowering potassium, but alone is inadequate to treat metabolic acidosis and often induces metabolic acidosis because of the removal of bicarbonate in the ultrafiltrate. In combination with $8.4 \%$ sodium bicarbonate, Z-BUF-S is highly effective at treating both hyperkalemia and metabolic acidosis, and prior to the sodium bicarbonate shortage, was our standard method of performing Z-BUF. In our experience, this combination resulted in the utilization of over $150 \mathrm{mEq}$ of sodium bicarbonate per surgical case. In the presence of a sodium bicarbonate shortage, our institutional allotment of sodium bicarbonate could not support continuation of this protocol. Hence, in response to this shortage, we chose to use bicarbonate-containing dialysate (NxStage ${ }^{\circ}$ RFP- $400^{\circ}$ and RFP-402 ${ }^{\circ}$ ) as our Z-BUF solution.

Two prior single patient case reports suggested that ZBUF-D could be used to correct the hyperkalemia and metabolic acidosis associated with $\mathrm{CPB}[5,6]$. Our retrospective analysis sought to further demonstrate the efficacy and feasibility of utilizing Z-BUF-D during the sodium bicarbonate shortage in a larger patient population.

Although NxStage ${ }^{\circ}$ RFP $-400^{\circ}$ and RFP- $402^{\circ}$ solutions are not FDA-approved for intravenous use, the utilization of these solutions with Z-BUF is consistent with their use as replacement fluid during continuous renal replacement therapy, for which they are FDA-approved. Similar dialysate solutions commercially available include Duosol $^{\circ}$ (Braun, Bethlehem, PA) and Prismasate (Baxter, Deerfield, IL). Alternative replacement fluids (Lactated Ringer's and PlasmaLyte A) to dialysate were considered. The composition of these crystalloids all vary considerably when compared to the dialysate solutions (Table 1). We ultimately decided that dialysate was a better option because it was available as a zero-potassium solution and it contained bicarbonate rather than acetate or lactate, both of which would require hepatic metabolism to be converted to bicarbonate, which we felt could be highly variable during CPB.

The use of dialysate as the replacement fluid in Z-BUF resulted in similar changes in the serum bicarbonate compared to patients undergoing Z-BUF with $\mathrm{NaCl}$ as the replacement fluids. However, the use of dialysate in Z-BUF allowed our team to use over $100 \mathrm{mEq}$ less of intravenous sodium bicarbonate per patient; a critical amount during the bicarbonate shortage. Although the mean change in whole blood potassium was greater in patients undergoing Z-BUF-S, the cohort who did Z-BUF-D did meet our goals for potassium reduction. We postulate that the lower preZ-BUF potassium level in the Z-BUF-D cohort reflects the use of Z-BUF-D for the management of metabolic acidosis without significant hyperkalemia. Prior to the sodium bicarbonate shortage, patients with metabolic acidosis, but without hyperkalemia, would have been managed with $8.4 \%$ sodium bicarbonate without Z-BUF.

The reduction in bicarbonate use was instrumental in our institution's ability to manage the sodium bicarbonate shortage. Although some of the reduction in bicarbonate usage may be related to greater awareness of the usage of sodium bicarbonate by the providers, we believe that the use of dialysate has played a major role in our ability to manage patients undergoing $\mathrm{CPB}$. We do not believe that the use of dialysate had any adverse effects on patients. We have included patient characteristics and outcomes of patients undergoing Z-BUF-S in the 150 days prior to our conversion. The Z-BUF-S group is a sample of convenience and is included to provide the reader with an idea of our prior standard of care. Although the use of a sample of convenience limits our ability to draw direct conclusions, randomization, or even a matched cohort comparison, was not practical for this descriptive study. Despite this limitation, Z-BUF with dialysate appears to have similar outcomes to those seen with $\mathrm{NaCl}$ while utilizing significantly less sodium bicarbonate per surgery ( $52 \mathrm{mEq}$ vs $159 \mathrm{mEq}$ ).

Our analyses has a variety of limitations. The retrospective nature of our data interpretation limits the ability to determine the reasoning by which providers determined who should receive Z-BUF as well as determine the amount of replacement fluid used. There was a lack of standardization between the Z-BUF-S and ZBUF-D cohorts, especially in terms of procedure type, aortic cross clamp time and cardiopulmonary bypass time. The cohorts only included those patients undergoing Z-BUF during a period of 150 days prior and following the beginning of sodium bicarbonate shortage and our institution beginning to use dialysate as the replacement fluid. Despite these limitations, this retrospective analysis serves to demonstrate the feasibility of utilizing dialysate as a replacement fluid during Z-BUF.

\section{Conclusion}

Z-BUF with dialysate replacement fluid appears to be aneffective therapy for the treatment of hyperkalemia and metabolic acidosis in patients undergoing $\mathrm{CPB}$. It appears to be an effective alternative to Z-BUF with $\mathrm{NaCl}$ and in our experience significantly decreases the need for $8.4 \%$ sodium bicarbonate in patients undergoing $\mathrm{CPB}$ with Z-BUF. 


\section{Abbreviations}

CPB: Cardiopulmonary bypass; NaCl: Sodium chloride; Z-BUF: Zero balance ultrafiltration

\section{Acknowledgements}

Not applicable.

\section{Authors' contributions}

RM analyzed and interpreted the collected data. LF provided information regarding the zero balance ultrafiltration procedure. All authors read and approved the final manuscript.

\section{Funding}

None.

\section{Availability of data and materials}

Available upon request.

\section{Ethics approval and consent to participate}

The University of Nebraska Medical Center Institutional Review Board approved this retrospective analysis.

\section{Consent for publication}

Not applicable.

\section{Competing interests}

The authors declare that they have no competing interests.

\section{Author details}

'Department of Internal Medicine, University of Nebraska Medical Center, 983040 Nebraska Medical Center, Omaha, NE 68198-3040, USA. ${ }^{2}$ Department of Clinical Perfusion, University of Nebraska Medical Center, Omaha, NE, USA. ${ }^{3}$ Department of Anesthesiology, University of Nebraska Medical Center, Omaha, NE, USA. ${ }^{4}$ Department of Surgery, University of Nebraska Medical Center, Omaha, NE, USA.

Received: 20 June 2019 Accepted: 2 September 2019

Published online: 10 September 2019

\section{References}

1. Alston RP, Cormack L, Collinson C. Metabolic acidosis developing during cardiopulmonary bypass is related to a decrease in strong ion difference. Perfusion. 2004;19:145-52.

2. Martin DP, Gomez D, Tobias JD, Schechter W, Cusi C, AT C, et al. Severe hyperkalemia during cardiopulmonary bypass: etiology and effective therapy. World J Pediatr Congenit Heart Surg. 2012;4(2):197-200.

3. Zhu X, Ji B, Wang G, Liu J, Long C. The effects of zero-balance ultrafiltration on postoperative recovery after cardiopulmonary bypass: a meta-analysis of randomized controlled trials. Perfusion. 2012;27(5):386-92.

4. Thompson CA. Sodium bicarbonate shortage found to affect hospitals' daily operations. Am J Health Syst Pharm. 2017;74(16).

5. Mick S, Hilberath J, Davidson M, FitzGerald D. Zero balance ultrafiltration for the correction of acute acidosis after a period of prolonged deep hypothermic circulatory arrest. Perfusion. 2011;27(1):9-11.

6. Heath M, Raghunathan K, Welsby I, Maxwell C. Using zero balance ultrafiltration with dialysate as a replacement fluid for hyperkalemia during cardiopulmonary bypass. J Extra Corpor Technol. 2014;46:262-6.

7. Myburgh J, Mythen M. Resuscitation fluids. N Engl J Med. 2013;369:1243-51.

8. PureFlow bicarbonate solution. (2017). http://www.nxstage.com/hcp/ products/pureflow-b-solution/. Accessed 28 August 2017.

\section{Publisher's Note}

Springer Nature remains neutral with regard to jurisdictional claims in published maps and institutional affiliations. 